\title{
Radiation Resistance of GaAs Structures
}

Chmill V.B., Chuntonov A.V., Smol A.V., Vorobiev A.P. Institute of High Energy Physics, Protvino, Russia.

Khludkov S.S., Koretski A.A., Potapov A.I., Tolbanov O.P. Siberian Institute for Physics and Technology, Tomsk, Russia.

Protvino 1996 


\section{Introduction}

Our previous studies [1] of the properties of GaAs pad detectors after irradiation in neutron fields and gammas have shown the high radiation hardness these detectors, manufactured in Tomsk. Several recent reports have shown, however, that the radiation hardness of GaAs detectors to charged hadron beams is much lower. In the following we try to analyse systematically the all experimental irradiation data that we have and to clarify this situation from experimental point of view.

\section{Description of Investigated Structures}

The test $p-\pi-\nu-n$ structures[1], were fabricated in the Siberian Institute for Physics and Technology, Tomsk, using GaAs grown by the Bridgman method of crystal growth in a magnetic field and industrial material produced by the Czochralski method. The samples were $200 \div 300 \mu \mathrm{m}$ thick plates of low-resistivity $n$-type GaAs with surface area $2 \times 2 \mathrm{~mm}^{2}$. On these plates, by means of liquid-phase epitaxy in the case of Tomsk laboratory material, a $\sim 150 \mu \mathrm{m}$ thick high-resistivity layer with resistivity $10^{7} \mathrm{Ohm} \cdot \mathrm{cm}$ was formed by doping the substrate material with iron. In the case of industrial substrates that we have used further in charge particles radiation tests, the $70 \div 150 \mu \mathrm{m}$ thick high-resistivity layer was prepared by indiffusion of chromium as a dopant. Inside the high-resistivity layer, by means of controlled doping, a $\pi-\nu$ junction was formed at a depth of $20 \div 70 \mu \mathrm{m}$. The contact layer was made of $n^{+}$or $p$-type GaAs. To evaluate the radiation resistance as a function of doping and compensation we have used the indus- 
trial material with a different primary electron concentration $6 \cdot 10^{16} \div 3 \cdot 10^{17} \mathrm{~cm}^{-} 3$. The irradiation was carried out in stages, with checks of the response of the samples to beta particles from a ${ }^{106} R u$ source at each stage. The procedure of these measurement can be found some where [1].

\section{Experimental results}

\subsection{Neutron irradiation}

Our first neutron irradiation (see Table 2.) of the GaAs samples was carried out at the proton linear accelerator in the Institute for High Energy Physics Protvino [2]. Four samples were exposed to neutron irradiation, two commercial samples and two fabricated in the Tomsk laboratory. In order to simulate more faithfully the actual conditions to be expected in practical use of the detectors, one sample of each type was irradiated under a reverse bias voltage of $300 \mathrm{~V}$.

The neutron dose was accumulated by the GaAs samples at a rate of $(4 \div 5) \cdot 10^{12} \mathrm{n} / \mathrm{cm}^{2}$ per hour. Fig.1 shows the experimental data for irradiated samples which were biased to $300 \mathrm{~V}$. The behaviour of the samples that had no bias voltage applied during the irradiation was similar, and is't shown here. As figure shows the GaAs structures which were compensated by chromium degraded much earlier: neutron fluence of $4 \cdot 10^{14} \mathrm{n} / \mathrm{cm}^{2}$ led to results similar to those for $1.2 \cdot 10^{15} \mathrm{n} / \mathrm{cm}^{2}$ for samples doped with iron.

The much lower radiation resistance of chromium doped samples can be explained by following. The samples processing have the concentration of chromium ten times less than in the case 


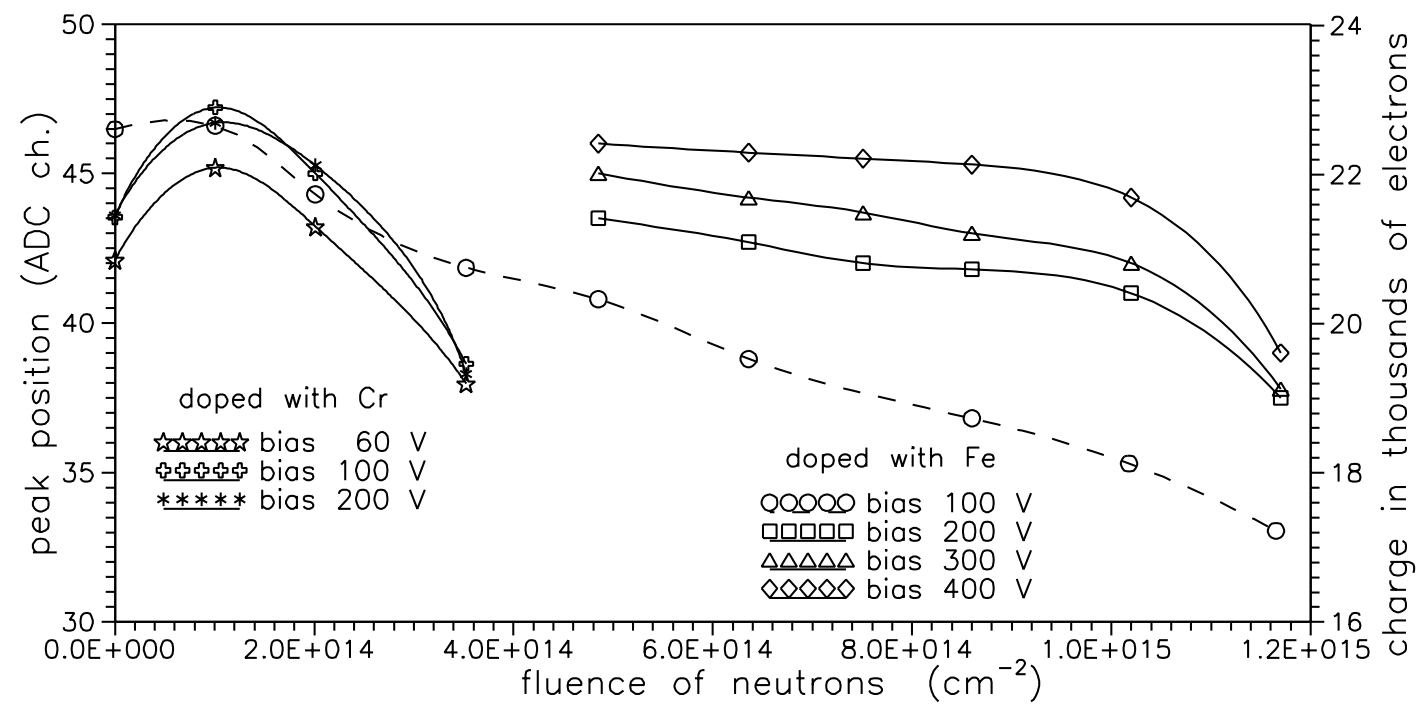

Figure 1: Neutron irradiation

of the iron dopping by the latter. But it is well known that radiation-induced defects in GaAs give deep trapping centers, which have compensating properties like iron and chromium. Consequently a higher concentration of the chromium or iron dopands at higher values of neutron fluence will have a greater influence over the radiative damages. The primary concentration of donors in chromium doped material, used in this test, was $3 \cdot 10^{16} \mathrm{~cm}^{-3}$.

The second round of neutron irradiation GaAs samples was carried out using Rutherford Appleton Laboratory Radiation Hardness Test Facility [3]. The samples have been irradiated at a rate of $(1.5 \div 3) \cdot 10^{12} \mathrm{n} / \mathrm{cm}^{2}$ per hour. The result presented in Table 1. for two samples $(N 110, N 111)$ with a primary donor concentration $(1 \div 2) \cdot 10^{17} \mathrm{~cm}^{-3}$ and the set of three detectors 
(N138) with donor concentration $(5 \div 6) \cdot 10^{16} \mathrm{~cm}^{-3}$. We are seeing again the indication to have higher radiation resistance for samples with large compensation level.

\begin{tabular}{|c|c|c|c|}
\hline Number & Structure & Fluence $\left[n / \mathrm{cm}^{2}\right]$ & Signal $\left[e^{-}\right]$ \\
\hline \hline $110-10-25$ & $n^{+}-\pi-\nu-n$ & Zero & $12^{\prime} 000$ \\
\hline & & $5 \cdot 10^{14}$ & $12^{\prime} 500$ \\
\hline & & $10^{15}$ & $10^{\prime} 400$ \\
\hline $111-7-1$ & $n^{+}-\pi-\nu-n$ & Zero & $7^{\prime} 800$ \\
\hline & & $5 \cdot 10^{14}$ & $12^{\prime} 000$ \\
\hline \hline $138 C 112_{2} C r / 2$ & & $10^{15}$ & $10^{\prime} 500$ \\
\hline & & Zero & $32^{\prime} 500$ \\
\hline \hline $138 C 112_{2} C r / 3$ & $n^{+}-\pi-\nu-n$ & $10^{15}$ & $19^{\prime} 500$ \\
\hline & & Zero & $28^{\prime} 000$ \\
\hline \hline $138 C 112_{2} C r / 6$ & $n^{+}-\pi-\nu-n$ & $10^{15}$ & $18^{\prime} 000$ \\
\hline & & Zero & $32^{\prime} 500$ \\
\hline
\end{tabular}

Table 1: Neutron irradiation at R.A.L.

\subsection{Proton irradiation}

The set of GaAs samples produced with industrial material have been irradiated in proton $1 \mathrm{GeV}$ beam of Protvino Booster accelerator in two runs. During the first run eight samples have been used with wide variation of the initial donor concentration. The proton dose was accumulated at a rate of $5 \cdot 10^{13} \mathrm{p} / \mathrm{cm}^{2}$ per hour. This rate is one order of magnitude higher than we had for neutron irradiation. The results of this run are presented in Fig.2, where we can see behaviour of the response of the samples to beta particles from $R u^{106}$ source. The samples withstand the accumulated dose of protons up to $2 \cdot 10^{14} \mathrm{p} / \mathrm{cm}^{2}$ with a less than 


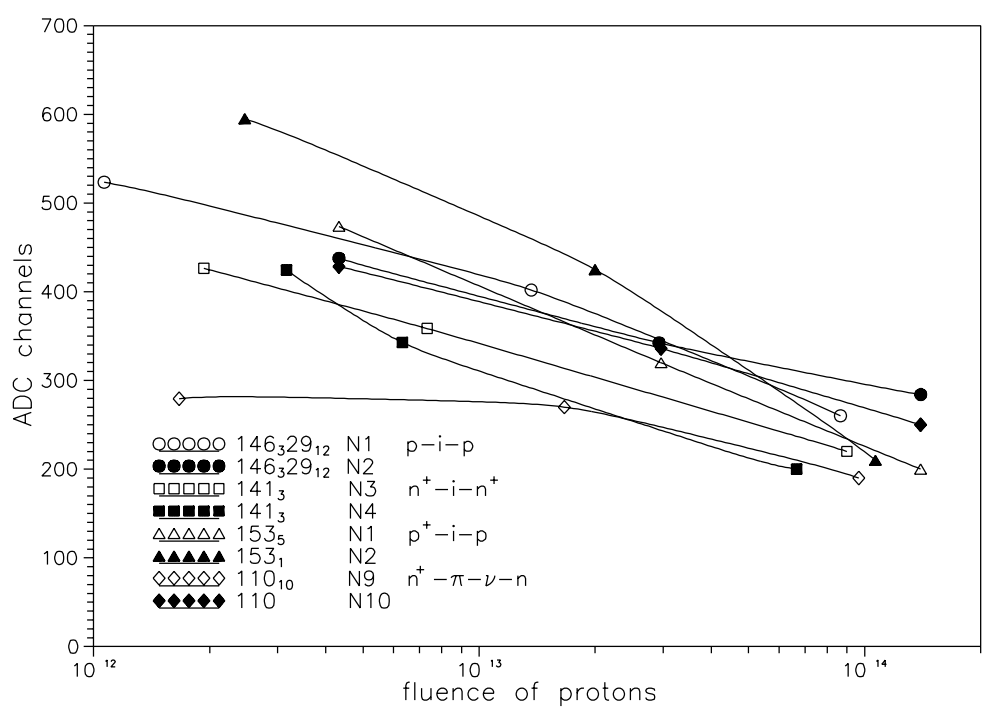

Figure 2: Proton irradiation, first run

$50 \%$ degradation of the signals. The second run with six samples was carried out with much higher irradiation rate $-5 \cdot 10^{14} \mathrm{p} / \mathrm{cm}^{2}$ per hour. We have lost the signals to beta particles somewhere between $10^{13} \div 10^{14} \mathrm{p} / \mathrm{cm}^{2}$ proton fluence. These results shown in Fig.3.

\subsection{Mixed beam irradiation}

The Tomsk GaAs detectors were installed this year together with Glasgow detectors in the West-Area CERN Neutrino Beam Facility where they were irradiated by the secondary beam from the T9 target. This beam contains mainly the charge particles in ratio $\left(\pi^{+}: \pi^{-}: K^{ \pm}: p\right) /(50: 14: 6: 30)$ and has a large part of neutrons, electrons and gammas. As we can estimate now from Monte Carlo simulation, the pions dose was accumulated 


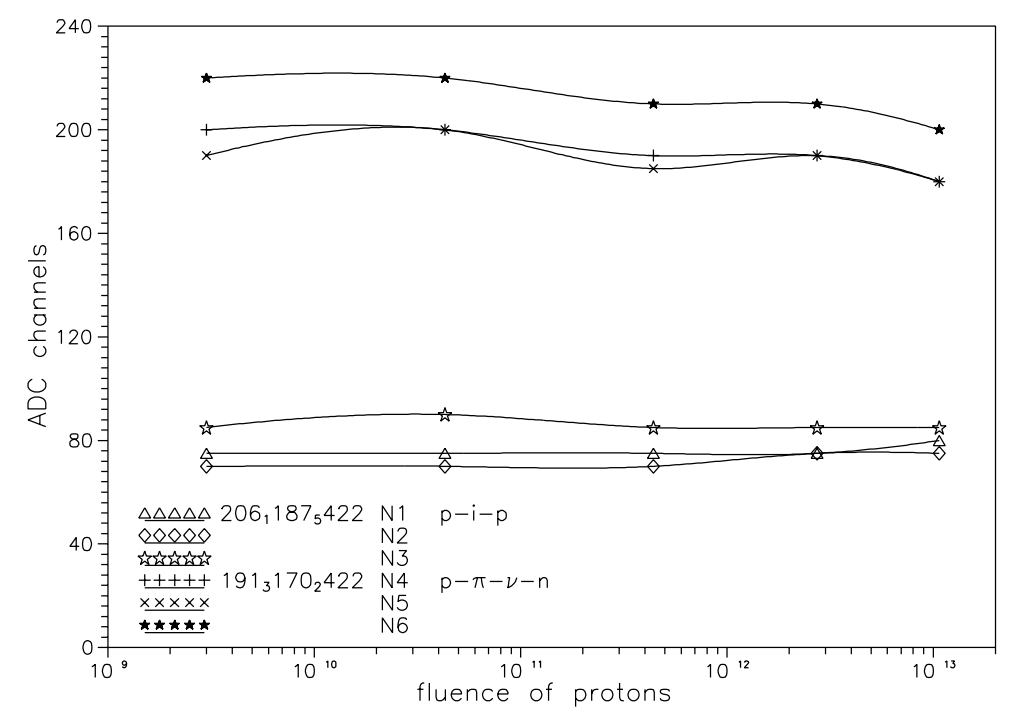

Figure 3: Proton irradiation, second run

by the samples at a rate of $2 \cdot 10^{12} \pi^{+} / \mathrm{cm}^{2}$ per hour. From our views this contain of the beam is the most near to the real use the GaAs detectors in the experiments, but the irradiation rate is still higher, than we will have in reality with microstrip detectors in collider experiment. In opposite to the previous cases we were measuring the response of GaAs detectors to incoming beam. The $20 \mathrm{~ms}$ base duration signal was flowing through $50 \mathrm{~m}$ long $50 \Omega$ cable from detectors directly to $1 M \Omega$ input impedance analog oscilloscope. The Fig.4 shows the values of pulse height corresponding to the average beam intensity. These measurements have been made without biasing of the detectors. Pulse height versus bias is presented in Fig.5 at three accumulated doses. A detailed description of this irradiation experiment using neutrino beam facility will be given elsewhere. 


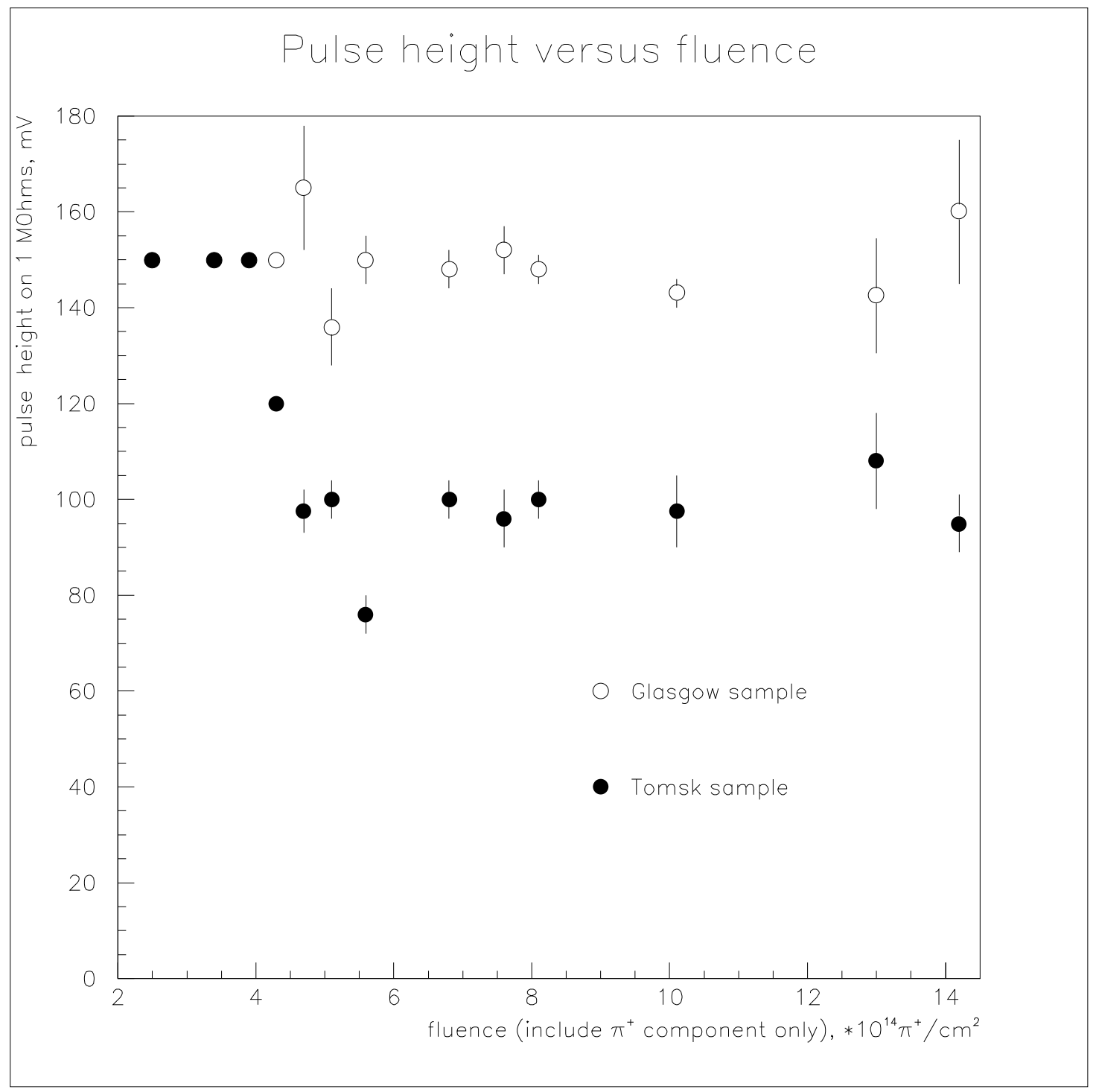

Figure 4: Pulse height vs. beam intensity. 


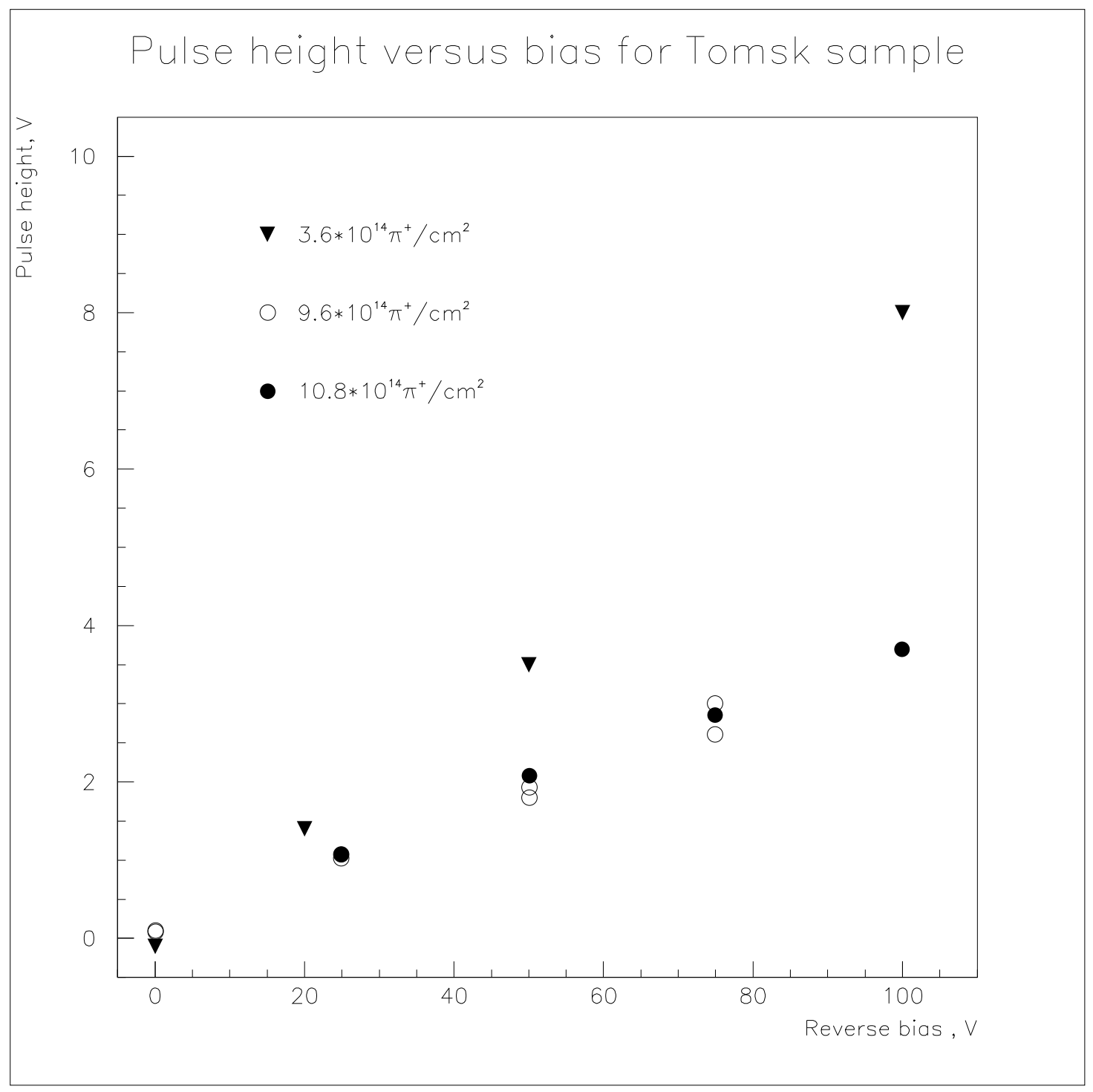

Figure 5: Pulse height for GaAs pad detector 
We summarized our results in the Table 2. The irradiation rate is one of the clue conditions in the evaluation of radiation hardness of the GaAs detectors in neutron and hadron beams. The radiation resistance of Tomsk detectors depends on the level of compensation. The manifestation of this fact can found, for example, in Fig.2 comparing the behaviour of signals versus fluence the samples $N 153$ with initial donor concentration $\left(1 \times 10^{16} \mathrm{~cm}^{-3}\right)$ and the samples $N 146$ with initial donor concentration $\left(2.5 \times 10^{16} \mathrm{~cm}^{-3}\right)$.

\begin{tabular}{|c|c|c|c|}
\hline Beam type & Accelerator & $\begin{array}{c}\text { Rate of irradiation } \\
\text { particles } \times \mathrm{cm}^{-2} \\
\text { per hour }\end{array}$ & $\begin{array}{c}\text { Final fluence } \\
\text { at } 50 \% \text { less } \\
\text { signal degradation }\end{array}$ \\
\hline $20 \mathrm{MeV}$ neutrons & Protvino I-100 & $(4 \div 5) \cdot 10^{12}$ & $1.2 \cdot 10^{15}$ \\
\hline $1 \mathrm{MeV}$ neutrons & R.A.L. ISIS & $(1.5 \div 3) \cdot 10^{12}$ & $1 \cdot 10^{15}$ \\
\hline $1 \mathrm{GeV}$ protons & Protvino Booster & $5 \cdot 10^{13}$ & $1.5 \cdot 10^{14}$ \\
\hline $\begin{array}{c}(0.8 \div 1) \mathrm{GeV} \\
\text { protons }\end{array}$ & Protvino Booster & $5 \cdot 10^{14}$ & $1.1 \cdot 10^{13}$ \\
\hline $\begin{array}{c}\text { Mixed beam } \\
\text { of neutrino facility }\end{array}$ & CERN SPS & $(2 \div 4) \cdot 10^{12}$ & $1.3 \cdot 10^{15}$ \\
\hline
\end{tabular}

Table 2: Summary result of hadron irradiation

\section{Conclusion}

GaAs structures based on commercial low resistivity materials with increased chromium doping concentration can be radiation hard. It can be expected that the radiation resistance of such structures will be similar to that of similar structures com- 
pensated by iron as was demonstrated by irradiation in a neutron beam. The present new results confirm the prospect using of such structures as a basis for the fabrication of radiationresistant coordinate-sensitive detectors. As seen in Table 2 the radiation hardness of GaAs structures depend on the irradiation rate and we conclude that the rate is the significant parameter in irradiation experiments. Detectors based on Tomsk structures we projected to have signals which will be degraded by less than $20 \%$ during 10 years of ATLAS operation.

\section{Acknowledgements}

We thank Prof. Kenway M. Smith and Dr. Saverio D'Auria for a careful reading of the manuscript and their help in preparing the most recently used experimental test beam area in CERN. We are most grateful Dr. Valery Falaleev for his help in preparing Monte Carlo calculation of beam contain and big help in equipment facility. We are also thankful to Dr. M. Edwards for his help with the irradiation of the detectors in R.A.L. 


\section{References}

[1] Vorobiev A.P. et. al. IHEP Preprint 91-192 Protvino, 1991 (in Russian).

Chmill V.B. et. al. Nucl. Instr. and Meth. V.A326, 1993, p.310.

Chmill V.B. et. al. Nucl. Instr. and Meth. V.A340, 1994, p.328-340.

[2] I-100 IHEP Preprint.

[3] Edwards M. and Perry D.R. Report RAL-90-065,1990 